\title{
Pairwise Comparison and Distance Measure of Hesitant Fuzzy Linguistic Term Sets
}

\author{
Han-Chen Huang ${ }^{1}$ and Xiaojun Yang ${ }^{2}$ \\ ${ }^{1}$ Department of Tourism and MICE, Chung Hua University, Hsinchu 30012, Taiwan \\ ${ }^{2}$ Luoyang Electronic Equipment Test Center, Luoyang, Henan 471003, China \\ Correspondence should be addressed to Xiaojun Yang; yangxiaojun2007@gmail.com
}

Received 10 April 2014; Accepted 3 July 2014; Published 4 August 2014

Academic Editor: Ker-Wei Yu

Copyright (C) 2014 H.-C. Huang and X. Yang. This is an open access article distributed under the Creative Commons Attribution License, which permits unrestricted use, distribution, and reproduction in any medium, provided the original work is properly cited.

\begin{abstract}
A hesitant fuzzy linguistic term set (HFLTS), allowing experts using several possible linguistic terms to assess a qualitative linguistic variable, is very useful to express people's hesitancy in practical decision-making problems. Up to now, a little research has been done on the comparison and distance measure of HFLTSs. In this paper, we present a comparison method for HFLTSs based on pairwise comparisons of each linguistic term in the two HFLTSs. Then, a distance measure method based on the pairwise comparison matrix of HFLTSs is proposed, and we prove that this distance is equal to the distance of the average values of HFLTSs, which makes the distance measure much more simple. Finally, the pairwise comparison and distance measure methods are utilized to develop two multicriteria decision-making approaches under hesitant fuzzy linguistic environments. The results analysis shows that our methods in this paper are more reasonable.
\end{abstract}

\section{Introduction}

Since Zadeh introduced fuzzy sets [1] in 1965, several extensions of this concept have been developed, such as type- 2 fuzzy sets $[2,3]$ and interval type-2 fuzzy sets [4], type- $n$ fuzzy sets [5], intuitionistic fuzzy sets $[6,7]$ and intervalvalued intuitionistic fuzzy sets [8], vague sets [9] (vague sets are intuitionistic fuzzy sets [10]), fuzzy multisets [11, 12], nonstationary fuzzy sets [13], Cloud models [14-18] (Cloud models are similar to nonstationary fuzzy sets and type-2 fuzzy sets), and hesitant fuzzy sets $[19,20]$. In the real world, there are many situations in which problems must deal with qualitative aspects represented by vague and imprecise information. So, in these situations, often the experts are more accustomed to express their assessments using linguistic terms rather than numerical values. In [2123], Zadeh introduced the concept of linguistic variable as "a variable whose values are not numbers but words or sentences in a natural or artificial language." Linguistic variable provides a means of approximate characterization of phenomena which are too complex or too ill defined to be amenable to description in conventional quantitative ways. Since then, fuzzy sets and linguistic variables have been widely used in describing linguistic information as they can efficiently represent people's qualitative cognition of an object or a concept [24]. Thus, linguistic approaches have been so far used successfully in a wide range of applications, such as information retrieval [25-28], data mining [29], clinical diagnosis $[30,31]$, and subjective evaluation [3237], especially in decision-making [38-49]. Usually, linguistic terms (words) are represented by fuzzy sets [50], type-2 fuzzy sets [51], interval type-2 fuzzy sets [52-54], 2-tuple linguistic model $[40,55]$, and so forth. In these linguistic models, an expert generally provides a single linguistic term as an expression of his/her knowledge. However, just as Rodriguez et al. [56] pointed out, the expert may think of several terms at the same time or look for a more complex linguistic term that is not defined in the linguistic term set to express his/her opinion. In order to cope with this situation, they recently introduced the concept of hesitant fuzzy linguistic term sets (HFLTSs) [56] under the idea of hesitant fuzzy sets introduced in $[19,20]$.

Similarly to a hesitant fuzzy set which permits the membership having a set of possible values, an HFLTS allows 
an expert hesitating among several values for a linguistic variable. For example, when people assess a qualitative criterion, they prefer to use a linguistic one such as "between medium and very high" which contains several linguistic terms \{medium, high, veryhigh\}, rather than a single linguistic term. In practical decision-making process, uncertainty and hesitancy are usually unavoidable problems. The HFLTSs can deal with such uncertainty and hesitancy more objectively, and thus it is very necessary to develop some theories about HFLTSs.

Comparisons and distance measures used for measuring the deviations of different arguments are fundamentally important in a variety of applications. In the existing literature, there are a number of studies on distance measures for intuitionistic fuzzy sets [57-60], interval-valued intuitionistic fuzzy sets [61], hesitant fuzzy sets [62, 63], linguistic values $[64,65]$, and so forth. Nevertheless, an HFLTS is a linguistic term subset, and the comparison among these elements is not simple. In [56], Rodriguez et al. introduced the concept of envelope for an HFLTS and then ranked HFLTSs using the preference degree method of interval values [66]. But, because an HFLTS is a set of discrete linguistic terms, it may seem problematical using the preference degree method for continuous interval to compare these discrete terms of HFLTSs. Up to now, just a few research has been done on the distance measure of HFLTSs [67]. Consequently, it is very necessary to develop some comparison methods and distance measure methods for HFLTSs. In [67], to calculate the distance of two HFLTSs, Liao et al. extend the shorter HFLTS by adding any value in it until it has the same length of the longer one according to the decision-maker's preferences and actual situations. In this paper, we present a new comparison method of HFLTSs based on pairwise comparisons of each linguistic term in the two HFLTSs. Then, a distance measure method based on the pairwise comparison matrix of HFLTSs is proposed without adding any value. Finally, we utilize the comparison method and distance measure method to develop some approaches to solve the multicriteria decision-making problems under hesitant fuzzy linguistic environments.

The rest of the paper is organized as follows. In Section 2, the concepts of hesitant fuzzy sets and HFLTSs are introduced; also the defects of the previous comparison method for HFLTSs are analyzed according to an example. Section 3 describes the comparison and distance measure of HFLTSs based on the proposed pairwise comparison method. In Section 4, a multicriteria decision-making problem is shown to illustrate the detailed processes and effectiveness of two ranking methods which are based on the comparisons and distance measures of HFLTSs, respectively. Finally, Section 5 draws our conclusions and presents suggestions for future research.

\section{Preliminaries}

2.1. Hesitant Fuzzy Sets. Hesitant fuzzy sets (HFSs) were first introduced by Torra [19] and Torra and Narukawa [20]. The motivation is that when determining the membership degree of an element into a set, the difficulty is not because we have a margin of error (such as an interval) but because we have several possible values.

Definition 1 (see [19]). Let $X$ be a fixed set; a hesitant fuzzy set (HFS) on $X$ is in terms of a function $h$ that when applied to $X$ returns a subset of $[0,1]$.

To be easily understood, Zhu et al. [68] represented the HFS as the following mathematical symbol:

$$
E=\{\langle x, h(x)\rangle \mid x \in X\}
$$

where $h(x)$ is a set of some values in $[0,1]$, denoting the possible membership degrees of the element $x \in X$ to the set $E$. Liao et al. [67] called $h(x)$ a hesitant fuzzy element (HFE).

Example 2. Let $h=\{0.2,0.3,0.4\}$; then $h$ is an HFE.

Definition 3 (see [69]). For an HFE $h$, the score function of $h$ is defined as

$$
s(h)=\frac{1}{\# h} \sum_{\gamma \in h} \gamma,
$$

where $\# h$ is the number of the elements in $h$.

For two HFEs $h_{1}$ and $h_{2}$, if $s\left(h_{1}\right)>s\left(h_{2}\right)$, then $h_{1}$ is superior to $h_{2}$, denoted by $h_{1}>h_{2}$; if $s\left(h_{1}\right)=s\left(h_{2}\right)$, then $h_{1}$ is indifferent with $h_{2}$, denoted by $h_{1} \sim h_{2}$.

Example 4. Assume that we have three HFEs, $h_{1}=$ $\{0.2,0.3,0.4\}, h_{2}=\{0.2,0.35,0.5\}$, and $h_{3}=\{0.3,0.4\}$; then according to the score function of HFE, (2), and Definition 3, we have $s\left(h_{1}\right)=(0.2+0.3+0.4) / 3=0.3, s\left(h_{2}\right)=(0.2+$ $0.35+0.5) / 3=0.35$, and $s\left(h_{3}\right)=(0.3+0.4) / 2=0.35$. Thus, $s\left(h_{2}\right)=s\left(h_{3}\right)>s\left(h_{1}\right)$; that is, the ranking is $h_{2} \sim h_{3}>h_{1}$.

The concept of HFS is very useful to express people's hesitancy in daily life. So, since it was introduced, more and more decision-making theories and methods under hesitant fuzzy environment have been developed [56, 62, 63, 67-73].

2.2. Hesitant Fuzzy Linguistic Term Sets. Similarly to the HFS, an expert may hesitate among several linguistic terms, such as "between medium and very high" or "lower than medium," to assess a qualitative linguistic variable. To deal with such situations, Rodriguez et al. [56] introduced the concept of hesitant fuzzy linguistic term sets (HFLTSs).

Definition 5 (see [56]). Suppose that $S=\left\{s_{0}, \ldots, s_{g}\right\}$ is a finite and totally ordered discrete linguistic term set, where $s_{i}$ represents a possible value for a linguistic variable. An HFLTS, $H_{S}$, is defined as an ordered finite subset of the consecutive linguistic terms of $S$.

It is required that the linguistic term set $S$ should satisfy the following characteristics:

(1) the set is ordered: $s_{i}>s_{j}$, if and only if $i>j$;

(2) there is a negation operator: $\operatorname{Neg}\left(s_{i}\right)=s_{g-i}$. 
Example 6. Let $S$ be a linguistic term set, $S=\left\{s_{0}\right.$ : $\mathrm{n}$ (nothing), $s_{1}$ : vl (very low), $s_{2}: 1$ (low), $s_{3}: \mathrm{m}$ (medium), $s_{4}:$ h (high), $s_{5}:$ vh (very high), and $s_{6}:$ p (perfect) $\}$; two different HFLTSs might be $H_{S}^{1}=\left\{s_{1}: \mathrm{vl}, s_{2}: 1, s_{3}: \mathrm{m}\right\}$ and $H_{S}^{2}=\left\{s_{3}: \mathrm{m}, s_{4}: \mathrm{h}\right\}$.

Definition 7. One defines the number of linguistic terms in the HFLTS $H_{S}$ as the cardinality of $H_{S}$, denoted by $\left|H_{S}\right|$. In Example 6, $\left|H_{S}^{1}\right|=3$ and $\left|H_{S}^{2}\right|=2$.

Definition 8 (see [56]). The lower bound $H_{S_{-}}$and upper bound $H_{S_{+}}$of the HFLTS $H_{S}$ are defined as $H_{S_{-}}=\min \left\{s_{i} \mid\right.$ $\left.s_{i} \in H_{S}\right\}$ and $H_{S+}=\max \left\{s_{i} \mid s_{i} \in H_{S}\right\}$.

Definition 9 (see [56]). The envelope of the HFLTS $H_{S}$, $\operatorname{env}\left(H_{S}\right)$, is defined as the linguistic interval $\left[H_{S_{-}}, H_{s_{+}}\right]=$ $\left[\operatorname{Ind}\left(H_{S_{-}}\right), \operatorname{Ind}\left(H_{S_{+}}\right)\right]$, where Ind provides the index of the linguistic term; that is, $\operatorname{Ind}\left(s_{i}\right)=i$. In Example 6, env $\left(H_{S}^{1}\right)=$ $\left[s_{1}, s_{3}\right]=[1,3]$ and $\operatorname{env}\left(H_{S}^{2}\right)=\left[s_{3}, s_{4}\right]=[3,4]$.

Based on the definition of envelope, Rodriguez et al. [56] compare two HFLTSs using the comparison method between two numerical intervals introduced by Wang et al. [66].

Definition 10 (see [66]). Letting $A=\left[a_{1}, a_{2}\right]$ and $B=\left[b_{1}, b_{2}\right]$ be two intervals, the preference degree of $A$ over $B$ (or $A>B$ ) is defined as

$$
P(A>B)=\frac{\max \left(0, a_{2}-b_{1}\right)-\max \left(0, a_{1}-b_{2}\right)}{\left(a_{2}-a_{1}\right)+\left(b_{2}-b_{1}\right)}
$$

and the preference degree of $B$ over $A$ (or $B>A$ ) is defined as

$$
P(B>A)=\frac{\max \left(0, b_{2}-a_{1}\right)-\max \left(0, b_{1}-a_{2}\right)}{\left(a_{2}-a_{1}\right)+\left(b_{2}-b_{1}\right)} .
$$

Example 11. Let $H_{S}^{1}=\left\{s_{1}, s_{2}, s_{3}\right\}, H_{S}^{2}=\left\{s_{3}, s_{4}\right\}$, and $H_{S}^{3}=\left\{s_{5}, s_{6}\right\}$ be three different HFLTSs on $S$. According to Definition 9, we have $\operatorname{env}\left(H_{S}^{2}\right)=[1,3] \operatorname{env}\left(H_{S}^{2}\right)=[3,4]$, and $\operatorname{env}\left(H_{S}^{3}\right)=[5,6]$. The preference degrees calculated by Definition 10, (3), and (4) are

$$
\begin{array}{ll}
P\left(H_{S}^{1}>H_{S}^{2}\right)=0, & P\left(H_{S}^{2}>H_{S}^{1}\right)=1 ; \\
P\left(H_{S}^{1}>H_{S}^{3}\right)=0, & P\left(H_{S}^{3}>H_{S}^{1}\right)=1 ; \\
P\left(H_{S}^{2}>H_{S}^{3}\right)=0, & P\left(H_{S}^{3}>H_{S}^{2}\right)=1 .
\end{array}
$$

From Example 11 mentioned above, it can be observed that when we compare two HFLTSs using the preference degree method, there exist two defects as follows.

(1) The result $P\left(H_{S}^{2}>H_{S}^{1}\right)=1$ indicates that $H_{S}^{2}$ is absolutely superior to $H_{S}^{1}$. In fact, both $H_{S}^{1}$ and $H_{S}^{2}$ contain the linguistic term $s_{3}$. It means that the value of a linguistic variable may be equal in these two cases. Thus, it is unreasonable to say that $H_{S}^{2}$ is absolutely superior to $H_{S}^{1}$.

(2) The result $P\left(H_{S}^{3}>H_{S}^{1}\right)=P\left(H_{S}^{2}>H_{S}^{1}\right)=1$, meaning that when compared with $H_{S}^{1}$, the two HFLTSs $H_{S}^{2}$ and $H_{S}^{3}$ are identical. In fact, $H_{S}^{3}$ is more superior to $H_{S}^{1}$ compared to $H_{S}^{2}$ to $H_{S}^{1}$. Thus, using the preference degree method to compare HFLTSs may result in losing some important information.

Based on the analysis mentioned above, we think that it is not suitable to compare discrete linguistic terms in HFLTSs using the comparison method for continuous numerical intervals. By the definition of an HFLTS, we know that every linguistic term in it is a possible value of the linguistic information. And noting that, the two HFLTSs for comparing may have different lengths. So, when comparing two HFLTSs, it needs pairwise comparisons of each linguistic term in them.

\section{Comparison and Distance Measure of HFLTSs}

3.1. Distance between Two Single Linguistic Terms. Let $s_{i}, s_{j} \in$ $S$ be two linguistic terms. $\mathrm{Xu}$ [64] defined the deviation measure between $s_{i}$ and $s_{j}$ as follows:

$$
d\left(s_{i}, s_{j}\right)=\frac{|i-j|}{T}
$$

where $T$ is the cardinality of $S$; that is, $T=|S|$.

If only one preestablished linguistic term set $S$ is used in a decision-making model, we can simply consider [49, 65]: $d\left(s_{i}, s_{j}\right)=|i-j|=s_{|i-j|}$.

Definition 12. Letting $s_{i}, s_{j} \in S$ be two single linguistic terms, then we call

$$
d\left(s_{i}, s_{j}\right)=s_{i}-s_{j}=i-j
$$

the distance between $s_{i}$ and $s_{j}$.

The distance measure between $s_{i}$ and $s_{j}$ has a definite physical implication and reflects the relative position and distance between $s_{i}$ and $s_{j}$. If $d\left(s_{i}, s_{j}\right)=0$, then $s_{i}=s_{j}$. If $d\left(s_{i}, s_{j}\right)>0$, then $s_{i}>s_{j}$. If $d\left(s_{i}, s_{j}\right)<0$, then $s_{i}<s_{j}$.

Theorem 13. Letting $s_{i}, s_{j}, s_{k} \in S$ be three linguistic terms, then

(1) $d\left(s_{i}, s_{j}\right)=-d\left(s_{j}, s_{i}\right)$;

(2) $(|S|-1) \leq d\left(s_{i}, s_{j}\right) \leq(|S|-1)$;

(3) $d\left(s_{i}, s_{k}\right)=d\left(s_{i}, s_{j}\right)+d\left(s_{j}, s_{k}\right)$.

Proof. They are straightforward and thus omitted.

3.2. Comparison of HFLTSs. The comparison of HFLTSs is necessary in many problems, such as ranking and selection. However, an HFLTS is a linguistic term subset which contains several linguistic terms, and the comparison among HFLTSs is not simple. Here, a new comparison method of HFLTSs, which is based on pairwise comparisons of each linguistic term in the two HFLTSs, is put forward.

Definition 14. Letting $H_{S}^{1}$ and $H_{S}^{2}$ be two HFLTSs on $S$, then one defines the pairwise comparison matrix between $H_{S}^{1}$ and $H_{S}^{2}$ as follows:

$$
C\left(H_{S}^{1}, H_{S}^{2}\right)=\left[d\left(s_{i}, s_{j}\right)\right]_{\left|H_{S}^{1}\right| \times\left|H_{S}^{2}\right|}, \quad s_{i} \in H_{S}^{1}, s_{j} \in H_{S}^{2} .
$$


Remark 15. The number of linguistic terms in the two HFLTSs, $H_{S}^{1}$ and $H_{S}^{2}$, may be unequal; that is, $\left|H_{S}^{1}\right| \neq\left|H_{S}^{2}\right|$. To deal with such situations, usually it is necessary to extend the shorter one by adding the stated value several times in it $[62,63]$, while our pairwise comparison method does not require this step.

Remark 16. From Definition 14, we have $\left[C\left(H_{S}^{1}, H_{S}^{2}\right)\right]=$ $-\left[C\left(H_{S}^{2}, H_{S}^{1}\right)\right]^{T}$, where $T$ is the transpose operator of matrix.

Example 17. Let $H_{S}^{1}=\left\{s_{1}, s_{2}, s_{3}\right\}$ and $H_{S}^{2}=\left\{s_{2}, s_{3}, s_{4}, s_{5}\right\}$ be two HFLTSs on $S$. According to Definition 14, the comparison matrix $C$ between $H_{S}^{1}$ and $H_{S}^{2}$ is

$$
\begin{aligned}
& s_{1} \\
& s_{2} \\
& s_{3}
\end{aligned} \quad\left[\begin{array}{cccc}
s_{2} & s_{3} & s_{4} & s_{5} \\
-1 & -2 & -3 & -4 \\
0 & -1 & -2 & -3 \\
1 & 0 & -1 & -2
\end{array}\right] .
$$

Definition 18. Letting $C=C\left(H_{S}^{1}, H_{S}^{2}\right)$ be the pairwise comparison matrix between $H_{S}^{1}$ and $H_{S}^{2}$, the preference relations of $H_{S}^{1}$ and $H_{S}^{2}$ are defined as follows:

$$
\begin{aligned}
& p\left(H_{S}^{1}>H_{S}^{2}\right)=\frac{\left|\sum_{C_{m n}>0} C_{m n}\right|}{\#\left\{C_{m n}=0\right\}+\sum\left|C_{m n}\right|}, \\
& p\left(H_{S}^{1}=H_{S}^{2}\right)=\frac{\#\left\{C_{m n}=0\right\}}{\#\left\{C_{m n}=0\right\}+\sum\left|C_{m n}\right|}, \\
& p\left(H_{S}^{1}<H_{S}^{2}\right)=\frac{\left|\sum_{C_{m n}<0} C_{m n}\right|}{\#\left\{C_{m n}=0\right\}+\sum\left|C_{m n}\right|} .
\end{aligned}
$$

It is obvious that $p\left(H_{S}^{1}>H_{S}^{2}\right)+p\left(H_{S}^{1}=H_{S}^{2}\right)+p\left(H_{S}^{1}<\right.$ $\left.H_{S}^{2}\right)=1$. We say that $H_{S}^{1}$ is superior to $H_{S}^{2}$ with the degree of $p\left(H_{S}^{1}>H_{S}^{2}\right)$, denoted by $H_{S}^{1}>^{p\left(H_{S}^{1}>H_{S}^{2}\right)} H_{S}^{2} ; H_{S}^{1}$ is equal to $H_{S}^{2}$ with the degree of $p\left(H_{S}^{1}=H_{S}^{2}\right)$, denoted by $H_{S}^{1} \sim^{p\left(H_{S}^{1}=H_{S}^{2}\right)} H_{S}^{2}$; and $H_{S}^{1}$ is inferior to $H_{S}^{2}$ with the degree of $p\left(H_{S}^{1}<H_{S}^{2}\right)$, denoted by $H_{S}^{1} \prec^{p\left(H_{S}^{1}<H_{S}^{2}\right)} H_{S}^{2}$.

Considering Example 17, by Definition 18, (10), the preference relations of $H_{S}^{1}$ and $H_{S}^{2}$ were calculated as $p\left(H_{S}^{1}>\right.$ $\left.H_{S}^{2}\right)=1 / 22, p\left(H_{S}^{1}=H_{S}^{2}\right)=2 / 22$, and $p\left(H_{S}^{1}<H_{S}^{2}\right)=19 / 22$. Thus, the comparison results are $H_{S}^{1} \succ^{1 / 22} H_{S}^{2}, H_{S}^{1} \sim^{2 / 22} H_{S}^{2}$, and $H_{S}^{1}{ }^{19 / 22} H_{S}^{2}$.

\subsection{Distance Measure of HFLTSs}

Definition 19. Letting $C=C\left(H_{S}^{1}, H_{S}^{2}\right)$ be the pairwise comparison matrix between $H_{S}^{1}$ and $H_{S}^{2}$, the distance between $H_{S}^{1}$ and $H_{S}^{2}$ is defined as the average value of the pairwise comparison matrix:

$$
d\left(H_{S}^{1}, H_{S}^{2}\right)=\frac{1}{\left|H_{S}^{1}\right| \times\left|H_{S}^{2}\right|} \sum_{m=1}^{\left|H_{S}^{1}\right|\left|H_{S}^{2}\right|} \sum_{n=1}^{2} C_{m n} .
$$

Considering Example 17, one has $d\left(H_{S}^{1}, H_{S}^{2}\right)=(-18) /$ $(3 \times 4)=-1.5$.
To preserve all the given information, the discrete linguistic term set $S$ is extended to a continuous term set $\bar{S}=\left\{s_{\alpha}\right.$ | $\alpha \in[-q, q]\}$, where $q$ is a sufficiently large positive number. If $s_{\alpha} \in S$, then we call $s_{\alpha}$ an original linguistic term; otherwise, we call $s_{\alpha}$ a virtual linguistic term.

Remark 20. In general, the decision-maker uses the original linguistic terms to express his/her qualitative opinions, and the virtual linguistic terms can only appear in operations.

Definition 21. The average value of an HFLTS $H_{S}$ is defined as

$$
\operatorname{Aver}\left(H_{S}\right)=\frac{1}{\left|H_{S}\right|} \sum_{s_{i} \in H_{S}} s_{i}=\frac{1}{\left|H_{S}\right|} \sum_{s_{i} \in H_{S}} \operatorname{Ind}\left(s_{i}\right) \text {. }
$$

This definition is similar to the score function of an HFE, Definition 3.

Considering Example 17, we have Aver $\left(H_{S}^{1}\right)=s_{(1+2+3) / 3}=$ $s_{2}=2$, and $\operatorname{Aver}\left(H_{S}^{2}\right)=s_{(2+3+4+5) / 4}=s_{3.5}=3.5$.

Theorem 22. Letting $H_{S}$ be an HFLTS on $S$, then

$$
0 \leq H_{s^{-}} \leq \operatorname{Aver}\left(H_{S}\right) \leq H_{s+} \leq(|S|-1) .
$$

Proof. It is straightforward and thus omitted.

Theorem 23. Letting $H_{S}^{1}$ and $H_{S}^{2}$ be two HFLTSs on $S$, the distance between $H_{S}^{1}$ and $H_{S}^{2}$ defined by the average value of their pairwise comparison matrix is equal to the distance of the two average values of $H_{S}^{1}$ and $H_{S}^{2}$; that is, the distance between $H_{S}^{1}$ and $H_{S}^{2}$ can be easily obtained by

$$
d\left(H_{S}^{1}, H_{S}^{2}\right)=\operatorname{Aver}\left(H_{S}^{1}\right)-\operatorname{Aver}\left(H_{S}^{2}\right) .
$$

Proof. From Definitions 19 and 14, we have

$$
\begin{aligned}
d\left(H_{S}^{1}, H_{S}^{2}\right) & =\frac{1}{\left|H_{S}^{1}\right| \times\left|H_{S}^{2}\right|} \sum_{m=1}^{\left|H_{S}^{1}\right|\left|H_{S}^{2}\right|} \sum_{n=1} C_{m n} \\
& =\frac{1}{\left|H_{S}^{1}\right| \times\left|H_{S}^{2}\right|} \sum_{s_{i} \in H_{S}^{1}} \sum_{s_{j} \in H_{S}^{2}} d\left(s_{i}, s_{j}\right) \\
& =\frac{1}{\left|H_{S}^{1}\right| \times\left|H_{S}^{2}\right|} \sum_{s_{i} \in H_{S}^{1}} \sum_{s_{j} \in H_{S}^{2}}\left(s_{i}-s_{j}\right) \\
& =\frac{1}{\left|H_{S}^{1}\right| \times\left|H_{S}^{2}\right|}\left(\sum_{s_{i} \in H_{S}^{1}} \sum_{s_{j} \in H_{S}^{2}} s_{i}-\sum_{s_{i} \in H_{S}^{1}} \sum_{s_{j} \in H_{S}^{2}} s_{j}\right) \\
& =\frac{1}{\left|H_{S}^{1}\right| \times\left|H_{S}^{2}\right|}\left(\left|H_{S}^{2}\right| \times \sum_{s_{i} \in H_{S}^{1}} s_{i}-\left|H_{S}^{1}\right| \times \sum_{s_{j} \in H_{S}^{2}} s_{j}\right) \\
& =\frac{1}{\left|H_{S}^{1}\right|} \sum_{s_{i} \in H_{S}^{1}} s_{i}-\frac{1}{\left|H_{S}^{2}\right|} \sum_{s_{j} \in H_{S}^{2}} s_{j} \\
& =\operatorname{Aver}\left(H_{S}^{1}\right)-\operatorname{Aver}\left(H_{S}^{2}\right),
\end{aligned}
$$

which completes the proof of Theorem 23. 
Considering Example 17, we have $d\left(H_{S}^{1}, H_{S}^{2}\right)=$ $\operatorname{Aver}\left(H_{S}^{1}\right)-\operatorname{Aver}\left(H_{S}^{2}\right)=2-3.5=-1.5$. lary.

By Theorem 23, we can easily obtain the following corol-

Corollary 24. Letting $H_{S}^{1}, H_{S}^{2}$ and $H_{S}^{3}$ be three HFLTSs on $S$, then

(1) $(|S|-1) \leq d\left(H_{S}^{1}, H_{S}^{2}\right) \leq(|S|-1)$;

(2) $d\left(H_{S}^{1}, H_{S}^{2}\right)=-d\left(H_{S}^{2}, H_{S}^{1}\right)$;

(3) $d\left(H_{S}^{1}, H_{S}^{3}\right)=d\left(H_{S}^{1}, H_{S}^{2}\right)+d\left(H_{S}^{2}, H_{S}^{3}\right)$.

Proof. They are straightforward and thus omitted.

If $d\left(H_{S}^{1}, H_{S}^{2}\right)>0$ (or $\left.\operatorname{Aver}\left(H_{S}^{1}\right)>\operatorname{Aver}\left(H_{S}^{2}\right)\right)$, then we say that $H_{S}^{1}$ is superior to $H_{S}^{2}$ with the distance of $d\left(H_{S}^{1}, H_{S}^{2}\right)$, denoted by $H_{S}^{1} \stackrel{d\left(H_{S}^{1}, H_{S}^{2}\right)}{\succ} H_{S}^{2}$; if $d\left(H_{S}^{1}, H_{S}^{2}\right)=0\left(\operatorname{or} \operatorname{Aver}\left(H_{S}^{1}\right)=\right.$ Aver $\left(H_{S}^{2}\right)$ ), then we say that $H_{S}^{1}$ is indifferent to $H_{S}^{2}$, denoted by $H_{S}^{1} \sim H_{S}^{2}$; if $d\left(H_{S}^{1}, H_{S}^{2}\right)<0$ (or $\operatorname{Aver}\left(H_{S}^{1}\right)<\operatorname{Aver}\left(H_{S}^{2}\right)$ ), then we say that $H_{S}^{1}$ is inferior to $H_{S}^{2}$ with the distance of $d\left(H_{S}^{1}, H_{S}^{2}\right)$, denoted by $H_{S}^{1} \stackrel{d\left(H_{S}^{1}, H_{S}^{2}\right)}{<} H_{S}^{2}$.

\section{Multicriteria Decision-Making Models Based on Comparisons and Distance Measures of HFLTSs}

In this section, two new methods are presented for ranking and choice from a set of alternatives in the framework of multicriteria decision-making using linguistic information. One is based on the comparisons and preference relations of HFLTSs and the other is based on the distance measure of HFLTSs. We adopt Example 5 in [56] (Example 25 in our paper) to illustrate the detailed processes of the two methods.

Example 25 ([see [56]). Let $X=\left\{x_{1}, x_{2}, x_{3}\right\}$ be a set of alternatives, $C=\left\{c_{1}, c_{2}, c_{3}\right\}$ a set of criteria defined for each alternative, and $S=\left\{s_{0}:\right.$ n (nothing), $s_{1}:$ vl (very low), $s_{2}:$ l (low), $s_{3}:$ m (medium), $s_{4}: \mathrm{h}$ (high), $s_{5}:$ vh (very high), $s_{6}: \mathrm{p}$ (perfect) $\}$ the linguistic term set that is used to generate the linguistic expressions. The assessments that are provided in such a problem are shown in Table 1 and they are transformed into HFLTSs as shown in Table 2.

\subsection{Multicriteria Decision-Making Based on the Comparisons of HFLTSs}

Step 1. Considering each criterion $c_{i}(i=1,2,3)$, calculate the preference degrees between all the alternatives $x_{j}(j=$ $1,2,3)$.

Considering criterion $c_{1}, H_{S}^{x_{1}}=\left\{s_{1}, s_{2}, s_{3}\right\}, H_{S}^{x_{2}}=$ $\left\{s_{2}, s_{3}\right\}$ and $H_{S}^{x_{3}}=\left\{s_{4}, s_{5}, s_{6}\right\}$, so the preference degrees about criterion $c_{1}$ calculated using the comparison method of HFLTSs as described in Section 3.2 are $p c_{1}\left(x_{1}>x_{2}\right)=1 / 7$, $p c_{1}\left(x_{1}=x_{2}\right)=2 / 7, p c_{1}\left(x_{1}<x_{2}\right)=4 / 7 ; p c_{1}\left(x_{1}>x_{3}\right)=$ 0/27, $p c_{1}\left(x_{1}=x_{3}\right)=0 / 27, p c_{1}\left(x_{1}<x_{3}\right)=27 / 27 ; p c_{1}\left(x_{2}>\right.$ $\left.x_{3}\right)=0 / 15, p c_{1}\left(x_{2}=x_{3}\right)=0 / 15, p c_{1}\left(x_{2}<x_{3}\right)=15 / 15$.
TABLE 1: Assessments that are provided for the decision problem.

\begin{tabular}{cccc}
\hline & $c_{1}$ & $c_{2}$ & $c_{3}$ \\
\hline$x_{1}$ & Between vl and $\mathrm{m}$ & Between h and vh & $\mathrm{h}$ \\
$x_{2}$ & Between l and $\mathrm{m}$ & $\mathrm{m}$ & Lower than $\mathrm{l}$ \\
$x_{3}$ & Greater than $\mathrm{h}$ & Between vl and $\mathrm{l}$ & Greater than $\mathrm{h}$ \\
\hline
\end{tabular}

TABLE 2: Assessments transformed into HFLTSs.

\begin{tabular}{cccc}
\hline & $c_{1}$ & $c_{2}$ & $c_{3}$ \\
\hline$x_{1}$ & $\left\{s_{1}, s_{2}, s_{3}\right\}$ & $\left\{s_{4}, s_{5}\right\}$ & $\left\{s_{4}\right\}$ \\
$x_{2}$ & $\left\{s_{2}, s_{3}\right\}$ & $\left\{s_{3}\right\}$ & $\left\{s_{0}, s_{1}, s_{2}\right\}$ \\
$x_{3}$ & $\left\{s_{4}, s_{5}, s_{6}\right\}$ & $\left\{s_{1}, s_{2}\right\}$ & $\left\{s_{4}, s_{5}, s_{6}\right\}$ \\
\hline
\end{tabular}

Considering criterion $c_{2}, H_{S}^{x_{1}}=\left\{s_{4}, s_{5}\right\}, H_{S}^{x_{2}}=\left\{s_{3}\right\}$, and $H_{S}^{x_{3}}=\left\{s_{1}, s_{2}\right\}$, so the preference degrees about criterion $c_{2}$ calculated using the comparison method of HFLTSs as described in Section 3.2 are $p c_{2}\left(x_{1}>x_{2}\right)=3 / 3, p c_{2}\left(x_{1}=\right.$ $\left.x_{2}\right)=0 / 3, p c_{2}\left(x_{1}<x_{2}\right)=0 / 3 ; p c_{2}\left(x_{1}>x_{3}\right)=12 / 12$, $p c_{2}\left(x_{1}=x_{3}\right)=0 / 12, p c_{2}\left(x_{1}<x_{3}\right)=0 / 12 ; p c_{2}\left(x_{2}>x_{3}\right)=$ $3 / 3, p c_{2}\left(x_{2}=x_{3}\right)=0 / 3, p c_{2}\left(x_{2}<x_{3}\right)=0 / 3$

Considering criterion $c_{3}, H_{S}^{x_{1}}=\left\{s_{4}\right\}, H_{S}^{x_{2}}=\left\{s_{0}, s_{1}, s_{2}\right\}$, and $H_{S}^{x_{3}}=\left\{s_{4}, s_{5}, s_{6}\right\}$, so the preference degrees about criterion $c_{3}$ calculated using the comparison method of HFLTSs as described in Section 3.2 are $p c_{3}\left(x_{1}>x_{2}\right)=9 / 9$, $p c_{3}\left(x_{1}=x_{2}\right)=0 / 9, p c_{3}\left(x_{1}<x_{2}\right)=0 / 9 ; p c_{3}\left(x_{1}>x_{3}\right)=0 / 4$, $p c_{3}\left(x_{1}=x_{3}\right)=1 / 4, p c_{3}\left(x_{1}<x_{3}\right)=3 / 4 ; p c_{3}\left(x_{2}>x_{3}\right)=$ $0 / 36, p c_{3}\left(x_{2}=x_{3}\right)=0 / 36, p c_{3}\left(x_{2}<x_{3}\right)=36 / 36$.

Step 2. Aggregate the preference relations using the weighted average method: $p\left(x_{j}>x_{k}\right)=\operatorname{sum}\left(w_{i} \times p c_{i}\left(x_{j}>x_{k}\right)\right)$, $p\left(x_{j}=x_{k}\right)=\operatorname{sum}\left(w_{i} \times p c_{\mathrm{i}}\left(x_{j}=x_{k}\right)\right)$, and $p\left(x_{j}<x_{k}\right)=$ $\operatorname{sum}\left(w_{i} \times p c_{i}\left(x_{j}<x_{k}\right)\right)$, where $w_{i}$ is the weight of criterion $c_{i}$, and $\operatorname{sum}\left(w_{i}\right)=1$. In this paper, $w_{i}=1 / 3, i=1,2,3$. Thus, the final preference relations are $\left(x_{1}>x_{2}\right)=15 / 21, p\left(x_{1}=\right.$ $\left.x_{2}\right)=2 / 21, p\left(x_{1}<x_{2}\right)=4 / 21 ; p\left(x_{1}>x_{3}\right)=1 / 3$, $p\left(x_{1}=x_{3}\right)=1 / 12, p\left(x_{1}<x_{3}\right)=7 / 12 ; p\left(x_{2}>x_{3}\right)=1 / 3$, $p\left(x_{2}=x_{3}\right)=0, p\left(x_{2}<x_{3}\right)=2 / 3$.

Step 3. Rank the alternatives using the nondominance choice degree method as described in [56]. From the results of Step 2, it can be easily obtained that

$$
P_{D}^{S}=\left(\begin{array}{ccc}
- & \frac{11}{21} & 0 \\
0 & - & 0 \\
\frac{1}{4} & \frac{1}{3} & -
\end{array}\right)
$$

Thus, $\mathrm{NDD}_{1}=\min \{(1-0),(1-1 / 4)\}=3 / 4, \mathrm{NDD}_{2}=$ $\min \{(1-11 / 21),(1-1 / 3)\}=10 / 21$, and $\mathrm{NDD}_{3}=\min \{(1-$ $0),(1-0)\}=1$. Finally, the ranking of alternatives is $x_{3}>$ $x_{1}>x_{2}$. 
TABLE 3: Average values of the assessments.

\begin{tabular}{lccc}
\hline & $c_{1}$ & $c_{2}$ & $c_{3}$ \\
\hline$x_{1}$ & 2 & 4.5 & 4 \\
$x_{2}$ & 2.5 & 3 & 1 \\
$x_{3}$ & 5 & 1.5 & 5 \\
\hline
\end{tabular}

TABLE 4: Aggregation results of each alternative.

\begin{tabular}{llll}
\hline & $x_{1}$ & $x_{2}$ & $x_{3}$ \\
\hline Aggregation result & 3.5 & 2.2 & 3.8 \\
\hline
\end{tabular}

\subsection{Multicriteria Decision-Making Based on the Distance Measures of HFLTSs}

Step 1. Considering each criterion $c_{i}(i=1,2,3)$, calculate the average values of HFLTSs for all the alternatives $x_{j}(j=$ $1,2,3)$. The results are shown in Table 3.

Step 2. Aggregate the average values using the weighted average method. The results are shown in Table 4.

Step 3. Rank the alternatives using the distance measure method. Thus, the ranking of alternatives is $x_{3} \stackrel{0.3}{>} x_{1} \stackrel{1.3}{>} x_{2}$.

4.3. Results Analysis. In [56], the ranking of alternatives is $x_{1}>x_{3}>x_{2}$, while both methods in this paper are $x_{3}>x_{1}>x_{2}$. Note that the practical decision-making problem is quite different from other applications where wellestablished measures can be used to quantify the performance for validation. In decision-making, usually there is no ground truth data or quantitative measures to assess the performance of a method [37]. This is why "plausibility" is used rather than "validation." Here, we analyze the original assessments about each criterion of alternatives $x_{1}$ and $x_{3}$. Considering criterion $c_{1}$, the original assessments of $x_{1}$ and $x_{3}$ are "between $\mathrm{vl}$ and $\mathrm{m}$ " and "greater than h," respectively, so it is obviously $x_{3}>x_{1}$ about criterion $c_{1}$. Considering criterion $c_{2}$, the original assessments of $x_{1}$ and $x_{3}$ are "between $\mathrm{h}$ and vh" and "between vl and l," respectively, so this time $x_{1}>x_{3}$. Considering criterion $c_{3}$, the original assessments of $x_{1}$ and $x_{3}$ are " $\mathrm{h}$ " and "greater than $\mathrm{h}$," respectively, so $x_{3}>x_{1}$ again. Summarily, $x_{3}>x_{1}$ occurs twice, while $x_{1}>x_{3}$ only once. Thus, we believe that our result is more plausible.

\section{Conclusion}

The comparison and distance measure of HFLTSs are fundamentally important in many decision-making problems under hesitant fuzzy linguistic environments. From an example, we found that there existed two defects when comparing HFLTSs using the previous preference degree method. By analyzing the definition of an HFLTS, a new comparison method based on pairwise comparisons of each linguistic term in the two HFLTSs has been put forward. This comparison method does not need the assumption that the values in all HFLTSs are arranged in an increasing order and two
HFLTSs have the same length when comparing them. Then, we have defined a distance measure method between HFLTSs based on pairwise comparisons. Further, we have proved that this distance is equal to the distance of the average values of HFLTSs, which makes the distance measure much simpler. Finally, two new methods for multicriteria decision-making in which experts provide their assessments by HFLTSs have been proposed. The encouraging results demonstrate that our methods in this paper are more reasonable.

In the future, the application of HFLTSs to group decision-making problems will be explored. We will also investigate how to obtain the weights of criteria under hesitant fuzzy linguistic environments.

\section{Conflict of Interests}

The authors declare that there is no conflict of interests regarding the publication of this paper.

\section{References}

[1] L. A. Zadeh, "Fuzzy sets," Information and Control, vol. 8, pp. 338-353, 1965.

[2] M. Mizumoto and K. Tanaka, "Some properties of fuzzy sets of type 2," Information and Control, vol. 31, no. 4, pp. 312-340, 1976.

[3] J. M. Mendel and R. I. B. John, "Type-2 fuzzy sets made simple," IEEE Transactions on Fuzzy Systems, vol. 10, no. 2, pp. 117-127, 2002.

[4] J. M. Mendel and H. Wu, "Type-2 fuzzistics for symmetric interval type-2 fuzzy sets: part 1, forward problems," IEEE Transactions on Fuzzy Systems, vol. 14, no. 6, pp. 781-792, 2006.

[5] D. Dubois and H. Prade, Fuzzy Sets and Systems: Theory and Applications, Academic Press, New York, NY, USA, 1980.

[6] K. T. Atanassov, "Intuitionistic fuzzy sets," Fuzzy Sets and Systems, vol. 20, no. 1, pp. 87-96, 1986.

[7] K. T. Atanassov, "More on intuitionistic fuzzy sets," Fuzzy Sets and Systems, vol. 33, no. 1, pp. 37-45, 1989.

[8] K. T. Atanassov and G. Gargov, "Interval valued intuitionistic fuzzy sets," Fuzzy Sets and Systems, vol. 31, no. 3, pp. 343-349, 1989.

[9] W. Gau and D. J. Buehrer, "Vague sets," IEEE Transactions on Systems, Man and Cybernetics, vol. 23, no. 2, pp. 610-614, 1993.

[10] H. Bustince and P. Burillo, "Vague sets are intuitionistic fuzzy sets," Fuzzy Sets and Systems, vol. 79, no. 3, pp. 403-405, 1996.

[11] R. R. Yager, "On the theory of bags," International Journal of General Systems, vol. 13, no. 1, pp. 23-37, 1986.

[12] S. Miyamoto, "Remarks on basics of fuzzy sets and fuzzy multisets," Fuzzy Sets and Systems, vol. 156, no. 3, pp. 427-431, 2005.

[13] J. M. Garibaldi, M. Jaroszewski, and S. Musikasuwan, "Nonstationary fuzzy sets," IEEE Transactions on Fuzzy Systems, vol. 16, no. 4, pp. 1072-1086, 2008.

[14] D. Li, J. Han, X. Shi, and M. C. Chan, "Knowledge representation and discovery based on linguistic atoms," Knowledge-Based Systems, vol. 10, no. 7, pp. 431-440, 1998.

[15] D. Li, C. Liu, and W. Gan, "A new cognitive model: cloud model," International Journal of Intelligent Systems, vol. 24, no. 3, pp. 357-375, 2009. 
[16] X. Yang, L. Zeng, F. Luo, and S. Wang, "Cloud hierarchical analysis," Journal of Information \& Computational Science, vol. 7, no. 12, pp. 2468-2477, 2010.

[17] X. Yang, L. Zeng, and R. Zhang, "Cloud Delphi method," International Journal of Uncertainty, Fuzziness and KnowlegeBased Systems, vol. 20, no. 1, pp. 77-97, 2012.

[18] X. Yang, L. Yan, and L. Zeng, "How to handle uncertainties in AHP: the Cloud Delphi hierarchical analysis," Information Sciences, vol. 222, pp. 384-404, 2013.

[19] V. Torra, "Hesitant fuzzy sets," International Journal of Intelligent Systems, vol. 25, no. 6, pp. 529-539, 2010.

[20] V. Torra and Y. Narukawa, "On hesitant fuzzy sets and decision," in Proceedings of the IEEE International Conference on Fuzzy Systems, pp. 1378-1382, Jeju Island, Korea, August 2009.

[21] L. A. Zadeh, "The concept of a linguistic variable and its application to approximate reasoning-I," Information Sciences, vol. 3, pp. 199-249, 1975.

[22] L. A. Zadeh, "The concept of a linguistic variable and its application to approximate reasoning-II," Information Sciences, vol. 4, pp. 301-357, 1975.

[23] L. A. Zadeh, "The concept of a linguistic variable and its application to approximate reasoning-III," Information Sciences, vol. 9, no. 1, pp. 43-80, 1975.

[24] J. Ma, D. Ruan, Y. Xu, and G. Zhang, "A fuzzy-set approach to treat determinacy and consistency of linguistic terms in multicriteria decision making," International Journal of Approximate Reasoning, vol. 44, no. 2, pp. 165-181, 2007.

[25] G. Bordogna and G. Pasi, "A fuzzy linguistic approach generalizing boolean information retrieval: a model and its evaluation," Journal of the American Society for Information Science, vol. 44, no. 2, pp. 70-82, 1993.

[26] G. Bordogna and G. Pasi, "An ordinal information retrieval model," International Journal of Uncertainty, Fuzziness and Knowledge-Based Systems, vol. 9, supplement 1, 2001.

[27] J. Kacprzyk and S. Zadrozny, "Linguistic database summaries and their protoforms: towards natural language based knowledge discovery tools," Information Sciences, vol. 173, no. 4, pp. 281-304, 2005.

[28] E. Herrera-Viedma and A. G. López-Herrera, "A model of an information retrieval system with unbalanced fuzzy linguistic information," International Journal of Intelligent Systems, vol. 22, no. 11, pp. 1197-1214, 2007.

[29] H. Ishibuchi, T. Nakashima, and M. Nii, Classification and Modeling with Linguistic Information Granules: Advanced Approaches to Linguistic Data Mining, Springer, Berlin, Germany, 2004.

[30] R. Degani and G. Bortolan, "The problem of linguistic approximation in clinical decision making," International Journal of Approximate Reasoning, vol. 2, no. 2, pp. 143-162, 1988.

[31] E. Sanchez, "Truth-qualification and fuzzy relations in natural languages, application to medical diagnosis," Fuzzy Sets and Systems, vol. 84, no. 2, pp. 155-167, 1996.

[32] H. Lee, "Applying fuzzy set theory to evaluate the rate of aggregative risk in software development," Fuzzy Sets and Systems, vol. 79, no. 3, pp. 323-336, 1996.

[33] L. Martínez, "Sensory evaluation based on linguistic decision analysis," International Journal of Approximate Reasoning, vol. 44, no. 2, pp. 148-164, 2007.

[34] L. Martínez, J. Liu, D. Ruan, and J. Yang, "Dealing with heterogeneous information in engineering evaluation processes," Information Sciences, vol. 177, no. 7, pp. 1533-1542, 2007.
[35] J. Lu, Y. Zhu, X. Zeng, L. Koehl, J. Ma, and G. Zhang, "A linguistic multi-criteria group decision support system for fabric hand evaluation," Fuzzy Optimization and Decision Making, vol. 8, no. 4, pp. 395-413, 2009.

[36] R. de Andrés, M. Espinilla, and L. Martínez, "An extended hierarchical linguistic model for managing integral evaluation," International Journal of Computational Intelligence Systems, vol. 3, no. 4, pp. 486-500, 2010.

[37] D. Wu and J. M. Mendel, "Computing with words for hierarchical decision making applied to evaluating a weapon system," IEEE Transactions on Fuzzy Systems, vol. 18, no. 3, pp. 441-460, 2010.

[38] F. Herrera and E. Herrera-Viedma, "Choice functions and mechanisms for linguistic preference relations," European Journal of Operational Research, vol. 120, no. 1, pp. 144-161, 2000.

[39] F. Herrera, E. Herrera-Viedma, and L. Martínez, "A fusion approach for managing multi-granularity linguistic term sets in decision making," Fuzzy Sets and Systems, vol. 114, no. 1, pp. 4358,2000 .

[40] F. Herrera and L. Martínez, "A 2-tuple fuzzy linguistic representation model for computing with words," IEEE Transactions on Fuzzy Systems, vol. 8, no. 6, pp. 746-752, 2000.

[41] B. Arfi, "Fuzzy decision making in politics: a linguistic fuzzy-set approach," Political Analysis, vol. 13, no. 1, pp. 23-56, 2005.

[42] V. Huynh and Y. Nakamori, "A satisfactory-oriented approach to multiexpert decision-making with linguistic assessments," IEEE Transactions on Systems, Man, and Cybernetics B: Cybernetics, vol. 35, no. 2, pp. 184-196, 2005.

[43] D. Ben-Arieh and Z. Chen, "Linguistic group decision-making: opinion aggregation and measures of consensus," Fuzzy Optimization and Decision Making, vol. 5, no. 4, pp. 371-386, 2006.

[44] J. L. García-Lapresta, "A general class of simple majority decision rules based on linguistic opinions," Information Sciences, vol. 176, no. 4, pp. 352-365, 2006.

[45] J. Wang and J. Hao, "Fuzzy linguistic PERT," IEEE Transactions on Fuzzy Systems, vol. 15, no. 2, pp. 133-144, 2007.

[46] T. C. Wang and Y. H. Chen, "Applying fuzzy linguistic preference relations to the improvement of consistency of fuzzy AHP," Information Sciences, vol. 178, no. 19, pp. 3755-3765, 2008.

[47] Y. Dong, Y. Xu, and S. Yu, "Linguistic multiperson decision making based on the use of multiple preference relations," Fuzzy Sets and Systems, vol. 160, no. 5, pp. 603-623, 2009.

[48] P. D. Liu, "A novel method for hybrid multiple attribute decision making," Knowledge-Based Systems, vol. 22, no. 5, pp. 388-391, 2009.

[49] Y. Dong, W. Hong, Y. Xu, and S. Yu, "Selecting the individual numerical scale and prioritization method in the analytic hierarchy process: a 2-Tuple fuzzy linguistic approach," IEEE Transactions on Fuzzy Systems, vol. 19, no. 1, pp. 13-25, 2011.

[50] L. A. Zadeh, "Fuzzy logic = computing with words," IEEE Transactions on Fuzzy Systems, vol. 4, no. 2, pp. 103-111, 1996.

[51] J. M. Mendel, "Fuzzy sets for words: a new beginning," in Proceedings of the IEEE International conference on Fuzzy Systems, pp. 37-42, St. Louis, Mo, USA, May 2003.

[52] J. M. Mendel, "Computing with words and its relationships with fuzzistics," Information Sciences, vol. 177, no. 4, pp. 988-1006, 2007.

[53] F. Liu and J. M. Mendel, "Encoding words into interval type2 fuzzy sets using an interval approach," IEEE Transactions on Fuzzy Systems, vol. 16, no. 6, pp. 1503-1521, 2008. 
[54] S. Coupland, J. M. Mendel, and D. Wu, "Enhanced interval approach for encoding words into interval type-2 fuzzy sets and convergence of the word FOUs," in Proceedings of the 6th IEEE World Congress on Computational Intelligence (WCCI '10), pp. 1-8, Barcelona, Spain, July 2010.

[55] L. Martínez and F. Herrera, "An overview on the 2-tuple linguistic model for computing with words in decision making: extensions, applications and challenges," Information Sciences, vol. 207, pp. 1-18, 2012.

[56] R. M. Rodriguez, L. Martinez, and F. Herrera, "Hesitant fuzzy linguistic term sets for decision making," IEEE Transactions on Fuzzy Systems, vol. 20, no. 1, pp. 109-119, 2012.

[57] E. Szmidt and J. Kacprzyk, "Distances between intuitionistic fuzzy sets," Fuzzy Sets and Systems, vol. 114, no. 3, pp. 505-518, 2000.

[58] P. Grzegorzewski, "Distances between intuitionistic fuzzy sets and/or interval-valued fuzzy sets based on the Hausdorff metric," Fuzzy Sets and Systems, vol. 148, no. 2, pp. 319-328, 2004.

[59] W. Wang and X. Xin, "Distance measure between intuitionistic fuzzy sets," Pattern Recognition Letters, vol. 26, no. 13, pp. 20632069, 2005.

[60] Z. S. Xu and J. Chen, "An overview of distance and similarity measures of intuitionistic fuzzy sets," International Journal of Uncertainty, Fuzziness and Knowledge-Based Systems, vol. 16, no. 4, pp. 529-555, 2008.

[61] Z. S. Xu, "A method based on distance measure for intervalvalued intuitionistic fuzzy group decision making," Information Sciences, vol. 180, no. 1, pp. 181-190, 2010.

[62] Z. Xu and M. Xia, "Distance and similarity measures for hesitant fuzzy sets," Information Sciences, vol. 181, no. 11, pp. 2128-2138, 2011.

[63] Z. Xu and M. Xia, "On distance and correlation measures of hesitant fuzzy information," International Journal of Intelligent Systems, vol. 26, no. 5, pp. 410-425, 2011.

[64] Z. S. Xu, "Deviation measures of linguistic preference relations in group decision making," Omega, vol. 33, no. 3, pp. 249-254, 2005.

[65] Y. Xu and H. Wang, "Distance measure for linguistic decision making," Systems Engineering Procedia, vol. 1, pp. 450-456, 2011.

[66] Y. M. Wang, J. B. Yang, and D. L. Xu, "A preference aggregation method through the estimation of utility intervals," Computers and Operations Research, vol. 32, no. 8, pp. 2027-2049, 2005.

[67] H. Liao, Z. Xu, and X. Zeng, "Distance and similarity measures for hesitant fuzzy linguistic term sets and their application in multi-criteria decision making," Information Sciences, vol. 271, pp. 125-142, 2014.

[68] B. Zhu, Z. Xu, and M. Xia, "Hesitant fuzzy geometric Bonferroni means," Information Sciences, vol. 205, pp. 72-85, 2012.

[69] M. Xia and Z. Xu, "Hesitant fuzzy information aggregation in decision making," International Journal of Approximate Reasoning, vol. 52, no. 3, pp. 395-407, 2011.

[70] X. Gu, Y. Wang, and B. Yang, "A method for hesitant fuzzy multiple attribute decision making and its application to risk investment," Journal of Convergence Information Technology, vol. 6, no. 6, pp. 282-287, 2011.

[71] M. Xia, Z. Xu, and N. Chen, "Some hesitant fuzzy aggregation operators with their application in group decision making," Group Decision and Negotiation, vol. 22, no. 2, pp. 259-279, 2013.

[72] G. Wei, "Hesitant fuzzy prioritized operators and their application to multiple attribute decision making," Knowledge-Based Systems, vol. 31, pp. 176-182, 2012.
[73] B. Zhu, Z. Xu, and M. Xia, "Dual hesitant fuzzy sets," Journal of Applied Mathematics, vol. 2012, Article ID 879629, 13 pages, 2012. 


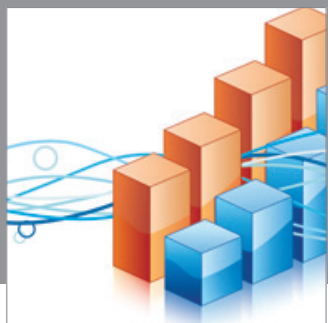

Advances in

Operations Research

mansans

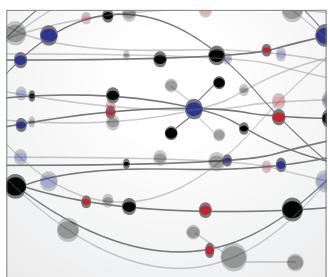

The Scientific World Journal
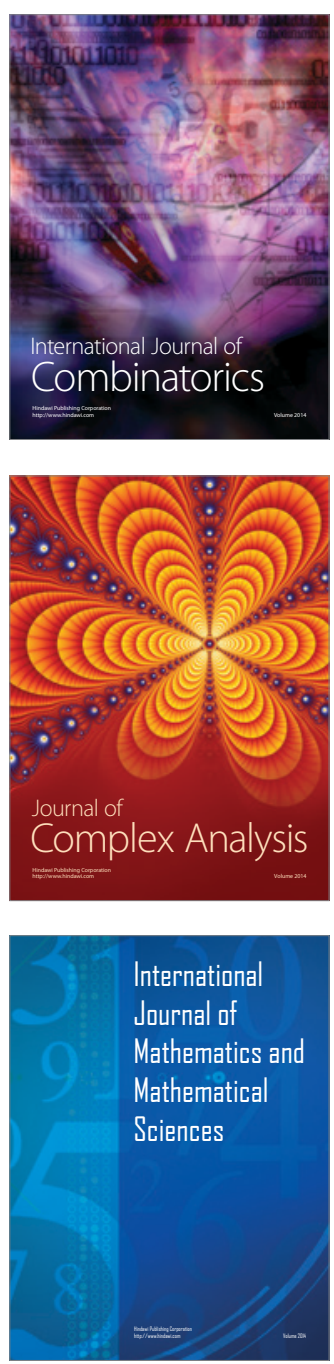
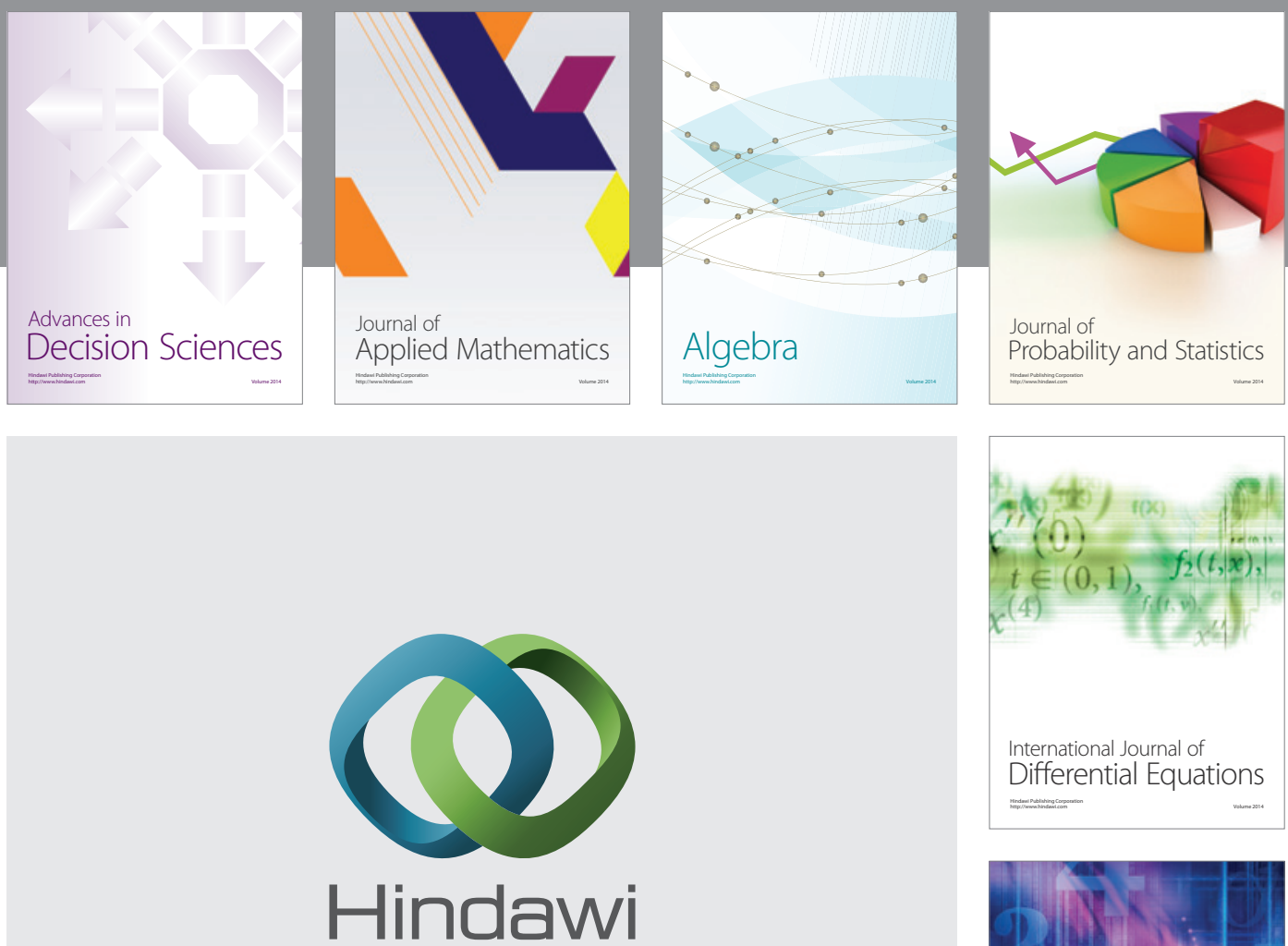

Submit your manuscripts at http://www.hindawi.com
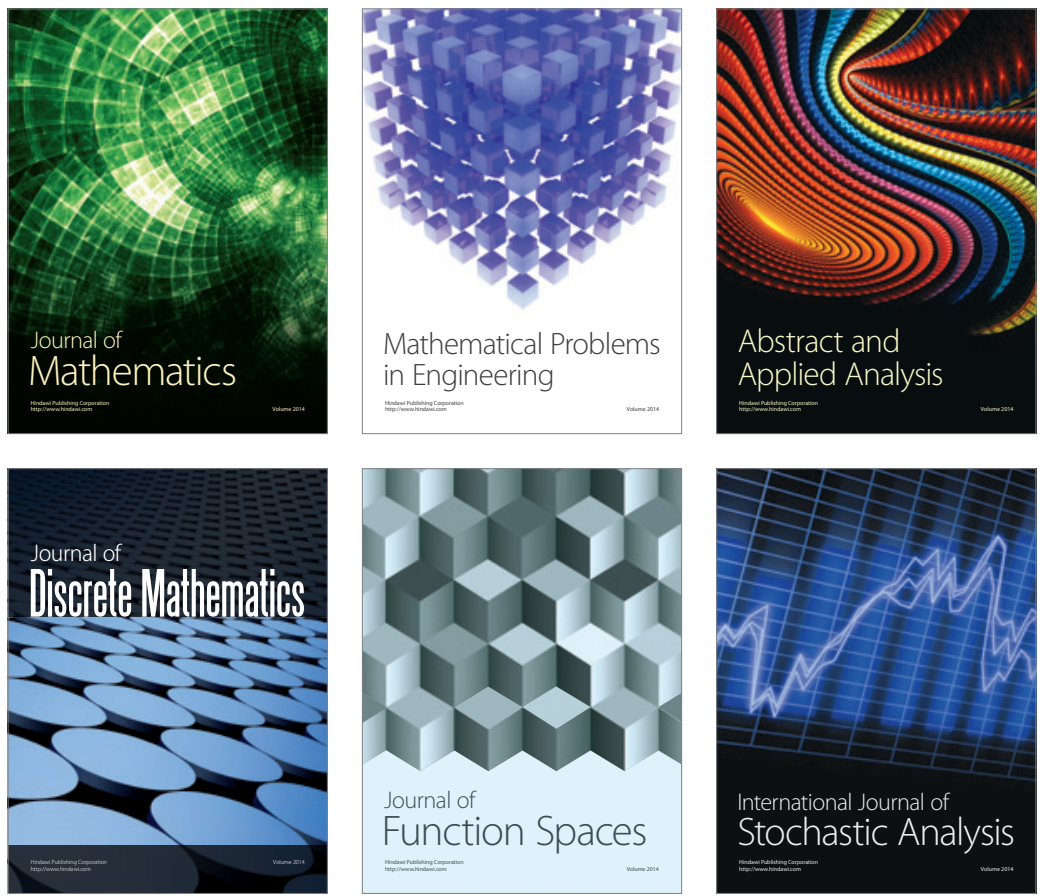

Journal of

Function Spaces

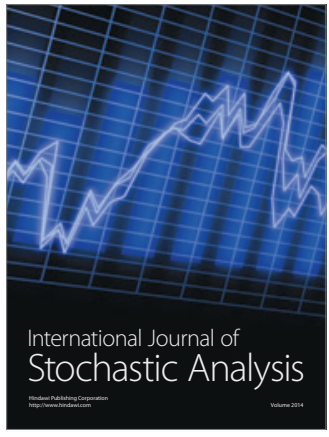

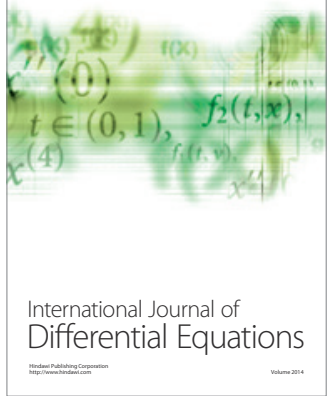
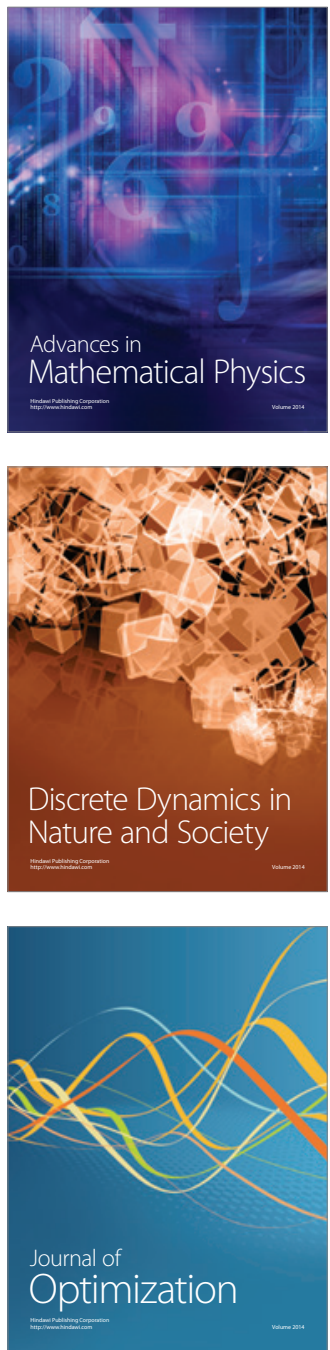